\title{
A new application for argon beam coagulation: the thymoma patient with pleural recurrence
}

\author{
Yen-Chiang Tseng ${ }^{1,2}$, Yen-Han Tseng ${ }^{3}$, En-Kuei Tang ${ }^{1}$, Yih-Gang Goan ${ }^{1}$ \\ ${ }^{1}$ Division of Thoracic Surgery, Department of Surgery, Kaohsiung Veterans General Hospital, Kaohsiung; ${ }^{2}$ Institute of Clinical Medicine, National \\ Yang-Ming University, Taipei; ${ }^{3}$ School of Medicine, National Yang-Ming University, Taipei \\ Correspondence to: Yih-Gang Goan, MD, PhD. Division of Thoracic Surgery, Department of Surgery, Kaohsiung Veterans General Hospital, No.386, \\ Dazhong 1st Rd., Zuoying Dist., Kaohsiung 81362. Email: yggoan@vghks.gov.tw.
}

\begin{abstract}
Thymomas are enigmatic tumors for which surgical resection is the mainstay of treatment. However, there are still many debates about resected thymomas with pleural recurrence. Repetitive operations for thymomas involving pleural recurrence are still the treatment of choice. Herein, we present a case with a suitable performance status for re-operation with a new application for argon beam coagulation. Both our experience and the currently available evidence suggest that surgical resection could be considered for patients with advanced thymomas, even for patients with locally advanced or Masaoka-Koga stage IV thymomas. Multimodality or multimodal, treatments resulted in better oncological outcomes for these patients. In this case, we proved that the new application of argon beam coagulation for a thymoma patient with pleural recurrence is safe and feasible. Additional evidence should be collected, and patients should be followed to assess long-term benefits.
\end{abstract}

Keywords: Argon beam coagulation; thymoma; pleural recurrence

Submitted Dec 07, 2017. Accepted for publication Mar 29, 2018.

doi: $10.21037 /$ jtd.2018.04.114

View this article at: http://dx.doi.org/10.21037/jtd.2018.04.114

\section{Introduction}

Thymoma is a rare and heterogeneous entity. Complete surgical resection has become the mainstay of treatment for low-grade diseases, while little data exist in the value and approach method of surgical resection in advanced stages (1). Re-operable disease was still considered to be a predictive factor for thymoma pleural recurrence (2). Nevertheless, how we could perform an immaculate resection with adequate safe margin of thymoma pleural recurrence remains controversial.

\section{Case presentation}

A 38-year-old female patient with myasthenia gravis, ocular type, presented to our clinic due to thymoma with pleural recurrence. The initial surgical intervention, extended thymectomy via median sternotomy, was performed in 2003. A Masaoka stage II, World Health Organization (WHO) histological type B1 encapsulated thymoma was confirmed. Adjuvant radiotherapy was added. However, during active surveillance, tumor recurrence from the right and left pleural cavities was noted sequentially. After video-assisted thoracic surgery (VATS) removal of visible tumors in 2008, WHO histological type B2/B3 thymoma gave rise to pleural seeding. All of the diagnostic slides were reviewed and confirmed by pathologists in our institution. Chemotherapy was administrated using cisplatin (CDDP) and etoposide for four cycles with good response. Unfortunately, recurrent soft tissues over left pleural cavity were detected by the positron emission tomography (PET) scan in 2012. Therefore, the adjuvant chemotherapy shifted to CDDP with etoposide for another 13 cycles was nearly complete response. However, subsequent tumor progression was noted 2 years later with chest computed tomography (Figure 1). After evaluation and discussion of multidisciplinary team, the stage IVA disease was diagnosed. Informed consent was obtained, and another VATS for tumor resection 

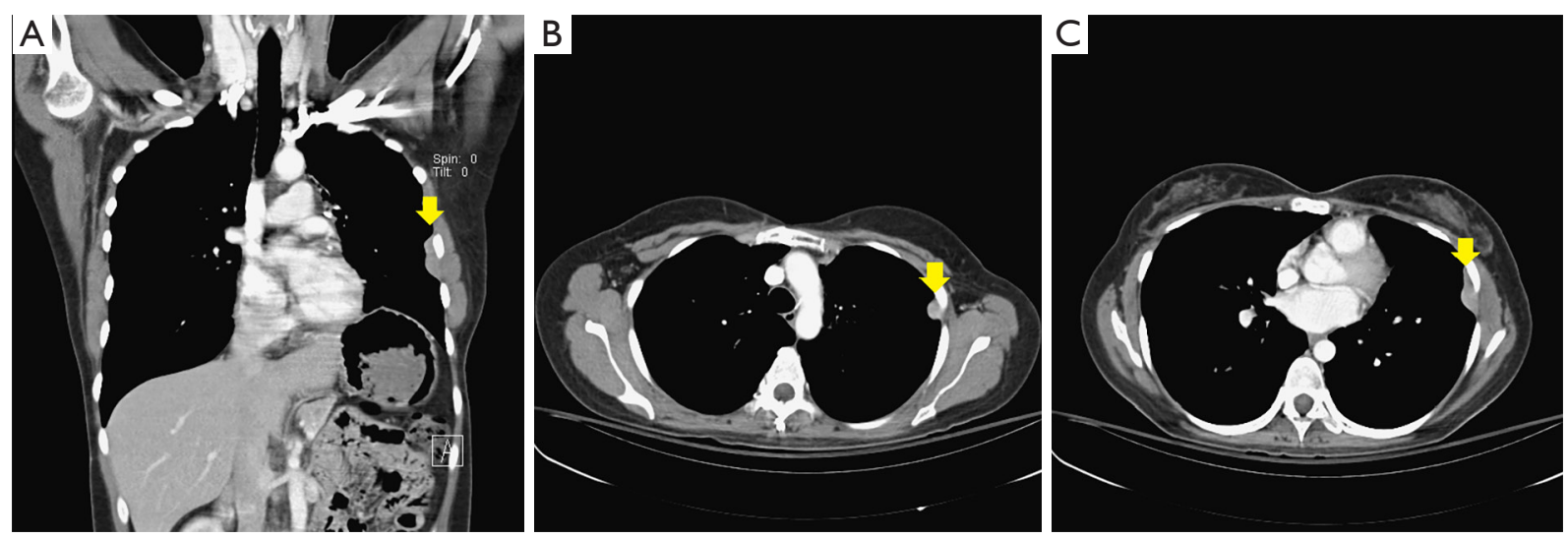

Figure 1 Chest computed tomography revealed thymoma pleural recurrence in left hemi-thorax. No matter in coronal section (A), or in axial view (B and C). Arrows, pleural tumor.
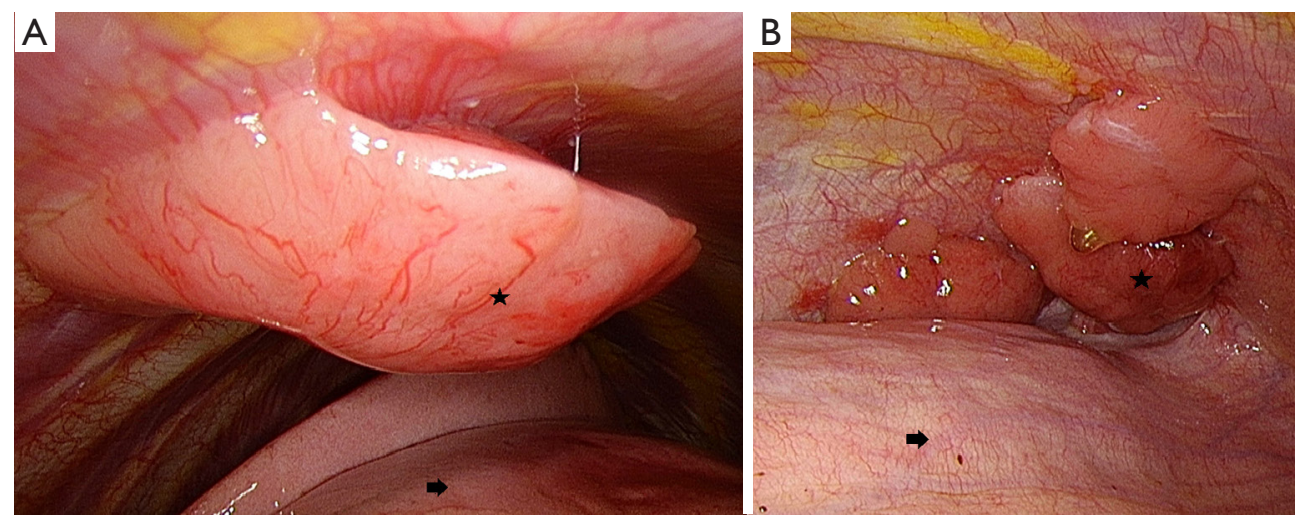

Figure 2 Under thoracoscopic examination, multiple pleural tumors with different sizes were found. The largest tumor measured $3 \mathrm{~cm}$ in length. Multiple small pleural tumors were found and were located at the costophrenic angle. (A) Arrow, lower left lobe; star, pleural tumor; (B) arrow, left diaphragm; star, tumor.

was arranged. Before surgical resection and argon beam coagulation, multiple pleural tumors of different sizes were found under thoracoscopic examination (Figure 2), which included more than ten implants in parietal pleura but no implant in visceral pleura. Most implants were in dependent part of left hemi-thorax, involved costophrenic angle and posterior lateral hemi-thorax. Surgical resection was initially in tumors with larger sizes, while argon beam coagulation was carried out for tumors with smaller sizes and for safe margins of all implants (Figure 3). The operation was performed smoothly and approximately $10 \mathrm{~mL}$ total blood loss. The 28 Fr straight chest tube was removed 7 days later. The patient was discharged smoothly on postoperative day 8 , who is by far under regular follow-up without any salient evidence of tumor recurrence. The exact follow up period after argon beam coagulation treatment was 6 months currently. Written informed consent was obtained from the patient for publication of this case report and any accompanying images.

\section{Discussion}

Thymoma is a mysterious, slowly progressing, and formidable endless disease. The incidence of thymic epithelial tumors is approximately 0.15 cases per year in the United States, which includes both thymoma and thymic carcinoma (3). Due to the rarity of thymomas, the clinical characteristics and indicators of prognosis are not well understood. Multidisciplinary collaboration among clinicians, surgeons, radiologists, and pathologists 

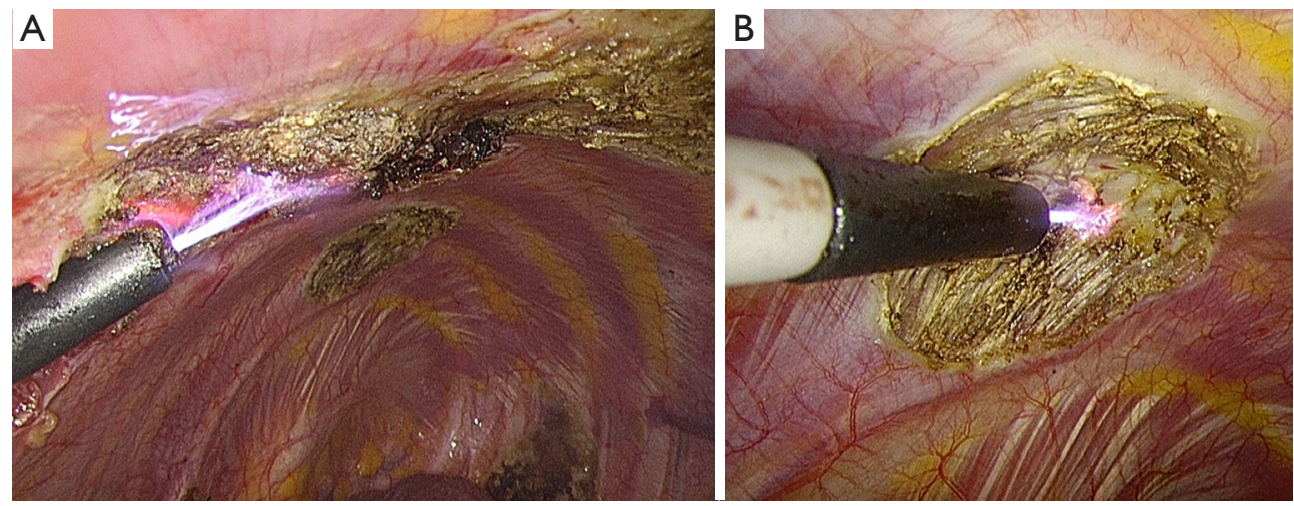

Figure 3 The argon beam coagulation with a curved probe could easily approach the tumor site.

is required to establish the diagnosis, to organize and implement the therapeutic strategy, and evaluate the prognosis (4). In non-myasthenic patients with earlystage thymomas, complete resection was the mainstay of treatment (5). However, there is scant evidence for the pros and cons of surgical resection of advanced stage thymomas or pleural tumor recurrence.

Surgery for thymomas with pleural recurrence is infrequently performed. Therefore, the value of surgical therapy for recurrent thymomas with pleural recurrence is deficiently defined. As mentioned previously, this case was identified as Masaoka stage IVA thymoma. Moser et al. collected the data from European Society of Thoracic Surgeons (ESTS) (6). They reported total 155 thymoma patients with pleural involvement, the percentages of primary intervention and surgery for recurrent disease are $70.4 \%$ and $29.6 \%$, respectively. Forty extrapleural pneumonectomies, 23 total pleurectomies, and 88 local pleurectomies were performed and similar survival rated was observed. Besides, incomplete resections predicted worse overall survival. Currently, this kind of patient faces a major challenge, we need to ensure complete re-resection under VATS since the possibility of potentially needing future procedures exists because of both frequent pleural and lung recurrences. Margaritora et al. pooled 18 studies published over a 17 -year period and found that 5 -year survival rates after re-resection ranged from $25 \%$ to $73 \%$. Completeness of resection was a major prognostic factor, similar to what is observed at the time of initial diagnosis, with 5 -year survival rates of $90 \%$ vs. $30 \%$ in the case of incomplete resection (7). Due to strenuous resection to a safe margin, Maury et al. reported intra-thoracic chemo-hyperthermia (ITCH) in addition to surgical cytoreduction (pleurectomy). Nineteen consecutive patients received surgical resection followed by ITCH with mitomycin and Cisplatin. ITCH provides long local control in selected patients. However, five patients presented with postoperative complication with three cases related to chemotherapy. Therefore, we still need to find a better modality for further local control for the patient with thymoma pleural recurrence (8).

In this case, we presented a new application for argon beam coagulation on account of the widespread application of minimally invasive surgery. In patients who have thymoma with pleural recurrence, tumor resection with conventional electrocoagulation was applied using posterolateral thoracotomy before (5). Regardless of surgical approach methods, a complete resection of thymoma pleural seeding with a better safety margin is necessary. The application of argon beam coagulation to control bleeding during liver resection is well documented (9). The principle, like that of all types of cautery, is the delivery of electrical energy to the tissue to cause tissue destruction. Highfrequency alternating current is delivered to the tissue via the flow of argon gas that has been ionized into electrically conductive plasma. As tissue is destroyed forming an eschar, conduction is lost preventing further damage and limiting the depth of tissue injury to $1-2 \mathrm{~mm}$. For this reason, its use is safer than that of techniques such as laser or conventional diathermy. Initially, argon beam coagulation was applied for the palliation of gastrointestinal tumors or hemostasis of liver surgery. We propose this new application because pleural recurrences of resected thymomas are prevalent and difficult to control. We tried this new application in an attempt to gain a better local and regional control over this 
aggressive, endless disease. However, additional evidence needs to be further obtained, and patients should be followed to assess long-term benefits.

\section{Acknowledgements}

None.

\section{Footnote}

Conflicts of Interest: The authors have no conflicts of interest to declare.

Informed Consent: Written informed consent was obtained from the patient for publication of this manuscript and any accompanying images.

\section{References}

1. Lucchi M, Davini F, Ricciardi R, et al. Management of pleural recurrence after curative resection of thymoma. J Thorac Cardiovasc Surg 2009;137:1185-9.

2. Tseng YH, Lin YH, Tseng YC, et al. Adjuvant Therapy for Thymic Carcinoma--A Decade of Experience in a Taiwan
National Teaching Hospital. PLoS One 2016;11:e0146609.

3. Engels EA, Pfeiffer RM. Malignant thymoma in the United States: demographic patterns in incidence and associations with subsequent malignancies. Int $\mathrm{J}$ Cancer 2003;105:546-51.

4. Engels EA. Epidemiology of thymoma and associated malignancies. J Thorac Oncol 2010;5:S260-5.

5. Tseng YC, Hsieh CC, Huang HY, et al. Is thymectomy necessary in nonmyasthenic patients with early thymoma? J Thorac Oncol 2013;8:952-8.

6. Moser B, Fadel E, Fabre D, et al. Surgical therapy of thymic tumours with pleural involvement: an ESTS Thymic Working Group Project. Eur J Cardiothorac Surg 2017;52:346-55.

7. Margaritora S, Cesario A, Cusumano G, et al. Singlecentre 40-year results of redo operation for recurrent thymomas. Eur J Cardiothorac Surg 2011;40:894-900.

8. Maury JM, Girard N, Tabutin M, et al. Intra-Thoracic Chemo-Hyperthermia for pleural recurrence of thymoma. Lung Cancer 2017;108:1-6.

9. Postema RR, Plaisier PW, ten Kate FJ, et al. Haemostasis after partial hepatectomy using argon beam coagulation. Br J Surg 1993;80:1563-5.
Cite this article as: Tseng YC, Tseng YH, Tang EK, Goan YG. A new application for argon beam coagulation: the thymoma patient with pleural recurrence. J Thorac Dis 2018;10(5):E355E358. doi: 10.21037/jtd.2018.04.114 